\title{
Towards an Ontology of Representation
}

\author{
Riichiro Mizoguchi ${ }^{\mathrm{a}}$ and Stefano Borgo ${ }^{\mathrm{b}}$ \\ ${ }^{a}$ Japan Advanced Institute of Science and Technology (JAIST), 1-1 Asahidai, Nomi, \\ Ishikawa 923-1292, Japan \\ E-mail:mizo@jaist.ac.jp \\ ${ }^{b}$ Laboratory for Applied Ontology, Institute of Cognitive Sciences and Technologies \\ (ISTC-CNR), Trento, Italy \\ E-mail: stefano.borgo@,cnr.it
}

\begin{abstract}
In philosophy information is mainly discussed along with the notion of aboutness. In more practical communities, information is mainly addressed together with notions like data and knowledge. This paper proposes a different approach. We look at information (and related concepts) as roles played by representations. This view implies that the notion of representation is central for any ontological analysis of information and related concepts. The paper provides arguments for this new stand and discusses an ontological model of representation based on the systematic distinction between form and content. The broadness and flexibility of the proposed model is shown by discussing a list of variegated representation entities from music to procedure, from novel to painting. The paper also investigates the role of letters (characters) in natural language expressions, which turns out to be quite complex.
\end{abstract}

Keywords. Representation, form, content, representing thing, specification, information

\section{Introduction}

Information is usually defined in ontology as a content-bearing entity that exists in reality [1]. This status of content-bearing follows from the assumption that the generated information is about what is observed, and the status of being about something is a problematic. Given this view, it is natural to take as a must for an ontological investigation of information to address the ontological status of aboutness [2][5], a notion that encounters important difficulties including the problem of 'fake' information (information which do not correspond to how things are in reality). One could argue that fake information is an epistemological issue and, if one takes the realist approach, this puts it out of scope of applied ontology. Yet, many consider this the sign of the inadequacy of our understanding of aboutness, and, in turn, of what information is.

In this paper we propose to take a different path. It claims that to understand information one must first understand representation. In other words, it says that in ontology representation is more fundamental than information. More cautiously, we observe that a theory of information presupposes a theory of representation and, thus, the latter must be developed to satisfactorily investigate the former. To support this view, one should note that information can be seen as a role played by a representation in the context of an event "to inform" (see Section 2). After all, information is borne in some type of representation, and some would even go as far as claiming that information itself must be borne to exist [1]. ${ }^{1}$

\footnotetext{
${ }^{1}$ An informal version of this approach was published in [3] [4].
} 
One can start from apparently naïve questions to expose the core of this issue. Consider, e.g., this question: "What is an instance of (a piece of) music?" Two answers come right to mind: it is a sequence of sounds (an event), and it is the score written by the composer or a copy of it. Music is a useful example in the discussion of representation because it seems immediate to the layman but formally it is hard to model. In the first case, namely, for music as the sound generated by instruments and players, a piece of music has nothing to do with representation. However, one could observe, the players performing the music follows a musical score (physically reading it or just remembering it by heart), and that musical score is a representation. ${ }^{2}$ From this observation, we can now deepen the initial answers about music. A 'piece of music' could refer to four distinct ontological entities:

(1) What the composer coded in musical language (the specification of the sound sequences the composer wants to characterize)

(2) The musical score (a text that has an associated semantics)

(3) The performance of the player (an activity)

(4) The sound generated by the performance (an event)

Let us now analyze the ontological status of these entities and the relationships between them. First, these four entities have clear relationships: the set of constraints/conditions (1) stated by the composer are captured by the semantics of the musical score (2) whose purpose is to make them explicit and shareable. The performance (3) is a realization process of the set of constraints such that the sound generated (4) in the performance is a realization of the sound patterns specified by them. Since the musical score, entity (2), is a representation in the ontological sense, our analysis will center around it. The reader should keep in mind that this is only an example and that our aim is not limited to music scores. Indeed, we aim to develop an ontology of representation that equally applies to procedures, recipes, dramas, novels, poems, paintings, car models, and the like. To be more precise, the notion of representation discussed in this paper covers the notions of depiction and of description; it does not include other representation meanings like "being appointed to act/speak for", "standing for" and so on. Briefly put, we investigate ontological representation objects, not rolebased representation objects as discussed, e.g., in semiotics.

We start with the assumption that there are language(s) and that they are endowed with semantics. These are the languages we care about. We also assume that these languages exist in time but not in space. They are created but not material in ontological argot. That is, we look at languages as set of rules (syntactical and semantical), not as sets of material marks. (One can be more demanding, for instance asking that these rules must always have a material, digital or neuronal support, but this issue is orthogonal to our work.) Note that our focus is on ontological aspects, not linguistic ones. Thus, we assume that these languages are not affected by ambiguity or, for what it matters, that any possible ambiguity has been already resolved when we discuss representations. Language can be symbolic, natural, analogical, iconic etc. including a mixture of these.

Given such a language, we take an ontological representation object to be an object that is necessarily composed by two ontological parts: an expression in that language (as said, not a material rendering of the expression), which we call form, and the meaning of that expression according to the language's semantics, which we call content. It follows that an ontological representation object exists in time but not in space,

\footnotetext{
${ }^{2}$ Improvisation in music, like in jazz sessions, is of course a separate issue and does not fall within the scope of this paper.
} 
as it is not a concrete entity not even in a parasitic sense like holes, and is independent of the existence of an agent that can understand or even recognize the object. To be precise, the relationship between a representation and the agents that understand, recognize or even create it is not part of the analysis we carry out (on the relationship between a representation and the human brain see, for instance, [1,2]). The relationship between form and content is called encoding. Here we do not discuss the specific characteristics of an encoding relation; it suffices to observe that it is usually based on (a combination of) the following: social conventions [7], private conventions (e.g., individual decisions of an agent), and similarity (e.g., the topological similarity between a subway map and a railroad network). This paper is about ontological representation objects whose encoding is based on social conventions. To avoid a possible misunderstanding, we clarify that encryption (as in cyphertext) is not a type of encoding as discussed in this paper. Indeed, an encryption is a relationship between forms of two languages (one of which may not even satisfy our definition of language since it does not need to have a semantics). From now on, we will use the term representation for ontological representation object.

Note that our notion of representation does not exclude "representation-of". It subsumes "representation-of". Consider a representation corresponding to sentence "John Smith is the third author of the paper". The representation, differently from the sentence, does not depend on the referred person John Smith, nor on what "the paper" is. Our representation theory does not aim to establish whether a representation is true or not, nor if there exists something in the world to which it refers, etc. Even representations that, taken as linguistic expressions, one may claim do not have a reference ("The French emperor is bald", "The sun rises from the West in Japan") are representations: they have form and content, and this is independent of what they are about (if anything). For another example, consider a procedure. A procedure is a representation; thus, it has a form and a content. It is important to understand that the content of a procedure is a specification for actions. A procedure does not refer to a/the/all/any procedure realization, and it is not about actions. Rather, it states the conditions a set of actions should satisfy to be a realization of that procedure. One can even give a procedure that is physically or even logically impossible to realize, it is a procedure to all effects since it has the needed form and content.

The paper is organized as follows. The next section discusses data, information, and knowledge, and presents these as roles played by a representation. It follows that representation is a fundamental concept for understanding them. Section 3 discusses form and content as components of representation. It also addresses the ways a representation can be realized, namely, the form-realization and the content-realization. Section 4 discusses the concept of representing thing: the concretization of a representation obtained by form-realization. In section 5 a preliminary formalization of representations and letters is presented to ease the understanding of the theory and to highlight dependences across the entities. Section 6 gives an overview of the entities introduced, adds considerations on the notions of copy and of identity of representation. It also discusses a different type of content needed to properly model the form of texts, paintings, etc. where form has a special value. Section 7 compares this approach to the IAO theory and other views. Section 8 adds the concluding remarks. 


\section{Data, Information and Knowledge as Representations}

There have been a lot of discussion about how to understand data, information and knowledge and their relationships [6] and people have tried to distinguish them primarily according to intrinsic properties and characteristics that may help to answer questions like "what is the difference between data and information?", "what can one do when she has knowledge vs. when she has data?" These attempts have not led to consensus [6]. Informally speaking, an expression like "Tokyo is the capital of Japan" becomes data when stored in a database, information when provided to another agent (human or artificial) who does not have it, and knowledge when an agent has learned it. While an appropriate discussion would take a full paper, we notice that all the discussions about data, information and knowledge address kinds of representations. This observation suggests that a representation becomes data, information or knowledge according to the context in which it is considered. This leads us to the following characterization:

a) Data is a representation when participating in an event in which it is processed, i.e., when the representation is the operand of a "to process" event. For instance, a person's name, a bare representation, is data when stored in a repository.

b) Information is a representation when participating in an event in which it is exchanged, i.e., when the representation is the operand of a "to inform" event. For instance, a person's name, a bare representation, is information when shown by a repository to an agent. Note that the status of being information is not related to the usefulness of the representation for some purpose, indeed there is no assumption that a purpose exists.

c) Knowledge is a representation when participating in an event in which it is learned (by an agent), i.e., when the representation is the operand of a "to learn" event. For instance, a person's name, a bare representation, is knowledge when an agent learns it (e.g., the agent can now use this representation to correctly address the person).

We acknowledge that these definitions may look limited. The stacks are high when discussing data, information, and knowledge, and any alternative view can easily look disappointing. However, at this stage we want only to highlight a general difference between the act of processing and the thing processed; the act of informing and the thing which is informed; the act of knowing (or learning) and the thing which is known (learned). While the thing processed, informed, or learned can be the same one, the acts are not. As suggested above, we look at data, information and knowledge as roles played by a representation in the context of processing, informing and learning/knowing. This view is grounded in the theory of roles presented in [10][11]. The assumption is that what people call data, information or knowledge is not intrinsically such. It follows that one needs to characterize the activities which provide a context in which these roles are defined. A first positive consequence of this choice is that it seems much easier to distinguish these acts, even though the specific understanding of each can vary from community to community. The confusing results in understanding data, information and knowledge [6] is a motivation for exploiting a different view which hopefully will turn out to be more precise and coherent. In conclusion, we have grounds to claim that representation should be investigated as an alternative foundation for the meaning of terms like data, information and knowledge. 


\section{On the Classification of Representations}

In the previous section we said that to make sense of data, information and knowledge one has to consider several ontological entities: activities, objects, roles and representations. In this section we show that our proposal is general enough to cover the variety of representation types. To do so, we consider seven distinct types of representation, namely: procedure, music, drama, novel, poem, handwriting (in calligraphy) and painting.

In the standard understanding of these types, all instances of the first five (procedure, music, drama, novel, poem) are created because of their content. A procedure's content is a specification of actions; a (piece of) music's content is a constraint/condition on sequences of sounds; a drama and novel's contents are sequences of happenings; a poem's content is a sequence of a variety of elements like scenes, thoughts, emotions. Handwriting and painting have content in a different way ${ }^{3}$, we can assume that the existence of these entities is primarily due to the form. We will come back to this issue below.

All these representations have authors/creators and users, and the first three also have performers, or so it is usually assumed. By authors, we mean agents who created the representation. By users, we mean agents who attend a performance (an event) based on the representation, for instance readers (in public reading events), audience or spectators. By performers, we mean agents that act following the representation content (thus, the public reading or recitation of a novel or poem does not count as performance and readers are not performers). For the first three, they can be human or artificial agents, musicians, and actors. The last four do not have performers. Those who at first sight seem to be performers are actually users, they are analogous to the listeners of music and spectators of drama. A poem recitation is sometimes considered a performance, it is not as we will see later. Note that users are not in the scope of our discussion because they do not characterize representations.

We said that representations are composed of two parts: form and content. By comparing different representation types, we can see that in some cases their reason to exist weights on one component more than the other. As for the first five types, the focus is clearly on the content which has only a generic dependence on the form. As to the last two, the form has a primary role to the point that the content is strictly dependent on the first: "the moral and philosophical implications in Chinese calligraphy [...] are associated directly with [...] the brush strokes and the way space is used, not merely derived from the general meaning of the words." (p.19, [8]). We already noticed that some representations, like music, are associated with two activity types: composing (creating) and performing. What can we say about painting? At first sight, a painter seems to be executing a composition (creation) act, as she is creating something anew as opposed to performing something according to specifications. It follows that music and painting are ontologically different representations: both have creators but only the first has performers.

Usually, one talks of realization as a mapping from a source to a destination, where the destination (the result of a realization process) is a concrete entity. As to the source, we limit ourselves to representations. Since a representation is composed of form and

\footnotetext{
${ }^{3}$ These two cases are sophisticated and are here treated somehow naively. They are considered mainly to show that our approach is applicable. Note that the meaning of the words or sentences in calligraphy is not important. A portrait has a content (the depicted person) which, we claim, is not important per sé.
} 
content, we can have form-realization and content-representation. The first may exist for all kinds of representation. Regarding content-realization, however, it may exist for procedures, pieces of music, and dramas only. In the case of the recitation of a poem, the source is essentially the form of the representation, so such a recitation act is not a performance. Although the content of a poem becomes relevant for other reasons (like artistic quality), it does not deal with the realization itself. When the content-realization exists, the representation content is called a specification. On the other hand, the form of a representation admits multiple realization types starting from: object realization (e.g., a sentence written on a sheet of paper) and process realization (e.g., speaking a sentence, reciting a song's lyrics). In sum, (1) procedure, piece of music and drama have both formrealization and content-realization; (2) novel, poem, handwriting (in calligraphy) and painting have form-realization but no content realization. Furthermore, the latter are obtained by a production activity as described below.

Consider engineering artifacts. They have two associated and clearly distinct phases, namely the design phase and the production phase. For what concerns us, these are two types of activities, they both have an outcome but of different type. We use design and production activities in artifacts to characterize the distinction between representation and realization in our theory. From this viewpoint a musical composition is a specification (a design, thus a set of rules) written in a language suitable for music, and a sequence of sounds satisfying the specification is an execution (a production, thus an object that satisfies the design). The composer, by creating music, produces a (material) musical score which is a form-realization of the musical composition (the composer realizes the design). The production corresponds to a content-realization in which a sequence of sounds is manufactured according to the design.

Up to this point we have talked of specifications only at the informal level taking them to be representations with realizable content. More precisely, we take a specification to be a consistent set of properties aimed to constrain an activity or its outcome (e.g., to guide the realization of an envisioned product). Ontologically speaking, a specification is an object (essentially a set of properties) created by an agent. Any object that satisfies the constraints is a realization of the specification. Any consistent set of properties (where consistent means that an entity satisfying those properties does not contradict natural laws) can be a specification provided it is intentionally chosen (created) by an agent at some point in time. In other words, given a specification, there exists a set of its realizations (the set may be empty when no realization has been produced). Vice versa, given a finite (non-empty) set of entities, one can generate a specification (usually, more than one) that includes the entities. When all and only the members of that set are realizations of the specification, there exists a class whose extension is the set and intension is the specification. Here are some consequences of the distinctions we have introduced.

\section{(1) Drama vs. Novel}

Drama and novel are ontologically different from the representation viewpoint. A drama is a specification and is realized by actors that perform the sequence of activities described in the drama, while a novel does not have such performance. Of course, readers read the novel, but reading does not count as performance. Instead, reading of a novel is comparable to attending (as spectator) a drama performance, listening to a musical sound sequence or watching a painting (the object). All these activities are not performances, they are perceptions. 
(2) Piece of music

A composer composes a specification of the sound sequence he/she creates. While there can be various performances of a piece of music, say, Beethoven's 5th Symphony (B5thS), and any two performances of that piece of music differ, all of them are understood as the B5thS because all the performances satisfy the rules coded in Beethoven's musical score (with some tolerance, of course). Given the set of all possible B5thS performances, Beethoven's piece of music is the commonality among all the performances, that is, a specification. What one listens to is a realization of the $\mathrm{B} 5 \mathrm{thS}$ and it is an instance of musical sound.

(3) Procedure

The same logic applies to procedure which is a specification. What the creator of a procedure creates, say, Hoar's quicksort algorithm, is an instance of procedure. The procedure is written down in a form (an expression in a suitable language) and such form may be realized on a sheet of paper ${ }^{4}$. The actual form depends on the chosen language but the content of the representation is the Quicksort algorithm. All the actions performed by following the Quicksort algorithm are realizations of the specification of Quicksort algorithm. Sometimes people talk of the running of the algorithm as an instance of it but, from our discussion, this way of speaking is ontologically wrong. The running of the algorithm is a realization of the Quicksort algorithm and an instance of a sorting action.

(4) Car model

The same applies to a car model by which we mean the content of a representation. The Prius car model is a specification. Mrs. Alice's Prius is an instance of a car and is a realization of the specification, i.e, of the Prius car model. A catalog of car models is a representing thing which may contain several car models as representations. We discuss representing things in the next section.

\section{Representing Thing}

Consider a published book. When we buy it in a bookstore we can say that we bought the book but, when pressed about what we mean, we clarify that we got a copy of the book. This leaves open how to ontologically understand the object that the author wrote. To answer this question, we need to understand the distinction between representation and representing thing. Recall that a representation exists in time but is not a concrete entity. A representation is created by an agent but is not material. This allows us to say that the sentence "This is a door" denotes two entities: on the one hand a representation that has form (in English) composed of a sequence of 11 things denoting alphabet letters (independently of the font or handwriting, i.e., of the patterns of the letter), on the other hand what one understands when reading "This is a door". Therefore, expression "This is a door" as a representation (an individual) is not a physical object (it does not exist in space). One can print (by assigning letters and fonts to the form), say on a sheet of paper, a series of "ink marks", we call this sequence of ink marks the representing thing of representation "This is a door". In this way the representation (the form and content pair) is associated with a physical individual that we call a representing thing. A representing thing is a form-realization and has two parts: the representation medium, namely, the material support (the sheet of paper with ink marks in this case) and the representation itself.

\footnotetext{
${ }^{4}$ More precisely, this is a representing thing as we will see later.
} 
The distinction between representation and representing thing enables us to distinguish the book that the author wrote, which is font-independent and thus a representation, from a representing thing, a copy of the book. The distinction between representation and representing thing is central and shows the importance of introducing non-physical entities in modeling representations. It also enables to make fine distinctions regarding the copies ${ }^{5}$ of a representation. What is copied is what one can perceive on the representation medium (in the case of a physical book, the medium is the sheet of paper). Copying is just a generation of the 'same' (as detailed in Sect. 6.3) representation on a different medium. In fact, a novel, say, Tale of Genji, exists in the form of a book. A copy of a book is a 3D entity physically divided in distinct pages (a complex medium) with the form realized in chapters, sections, etc. The content of the copy of the book is the content of the representation of the representing thing and is associated with the ink marks that represent the form expressions in natural language and/or images. In this sense we can say that the Tale of Genji exists independently of the medium on which it is written. The separation between representation and representing thing will be deepened in the following sections.

\section{A Formalization of the Ontology of Representations}

\subsection{Representation and Representing Thing}

In this section we provide a minimal characterization of the theory of representation as introduced in this paper. The aim of this section is to highlight the basic elements and their relationships, in particular relatively to notions like realization, sentence and pattern. This logical theory is also introduced with a modular perspective: the axiomatization should be aligned with the (possibly foundational) ontology one is using and further specialized depending on the characteristics of the latter. Note that the module is not seen as suitable for arbitrary ontologies as the theory relies on specific choices. In particular, it assumes that along the traditional notion of object there are semi-abstract entities. This is the case, for instance, of Yamato with the categories Physical and Semi-abstract, and of Dolce with the categories Physical Object (POB) and Non-Physical Object (NPOB). An example of semi-abstract entity, as understood here, is content. It is a non-material entity and it starts to exist at some point in time. Content is a special type of semi-abstract since it can be encoded, for instance in a sentence. A form, as understood in this paper, is also a semi-abstract.

A typical, yet complex, example of representation is given by natural language sentences. The form of a sentence in natural language encodes the meaning of the sentence as usual. Consider now a single letter. The form of the letter is a linear drawing (think of it as a non-localized image as to distinguish it from a representing thing) which encodes the standard pattern of the letter as its content. For a different example, in a sculpture the form is the 3D shape (again, not localized) which encodes the content of the sculpture. These forms are intrinsically 'spatial' because, even if not localized, they require spatial dimensions to exist. To understand these intrinsically spatial forms as semi-abstract, one can think of a digital representation in which a 2D or 3D image in the real world is captured in a digital form. This form can be realized on a display for human perception. In ontological terms, such digital representation is a specification of the $2 \mathrm{D}$ (or 3D) pattern. This specification is the form, and it is neither the content of the sentence

\footnotetext{
${ }^{5}$ This means not only photo-copies but also transcriptions.
} 
nor that of the statue. This form-specification is also not to be confused with the specification of, say, a procedure, which is the content of the procedure.

Coming back to individual letters (characters), the form of a letter as a specification is about the form-realization (the localizing of a letter pattern), while the content as a specification is about the content-realization (the drawing of a letter). Thus, in the case of letters the form-specification is a specification of the particular pattern of the letter to be localized, and that content-specification is a specification of the standard pattern of the letter as a specific element of the letter alphabet (in general a letter may be associated with more than one pattern, for simplicity here we assume there is only one).

First, we define representations as entities composed of two parts, a form and a content, connected by an encoding relationship with its related encoding method

$$
\begin{gathered}
\text { representation }(x, y, z, m)={ }_{\text {def }} \text { form }(y) \wedge \operatorname{content}(z) \wedge \operatorname{method}(m) \\
\wedge \text { encodes_via }(y, z, m) \wedge x=y+z
\end{gathered}
$$

The encoding relationship that we consider applies to form, content and method (in this order) and is functional

$$
\begin{aligned}
& (A x 1) \text { encodes_via }(x, y, z) \rightarrow \text { form }(x) \wedge \text { content }(y) \wedge \text { method }(z) \\
& (A x 2) \text { encodes_via }(x, y, z) \wedge \text { encodes_via }\left(x, y^{\prime}, z\right) \rightarrow y=y^{\prime} \\
& (A x 3) \text { encodes_via }(x, y, z) \wedge \text { encodes_via }\left(x^{\prime}, y, z\right) \rightarrow x=x^{\prime} \\
& (A x 4) \text { encodes_via }(x, y, z) \wedge \text { encodes_via }\left(x, y, z^{\prime}\right) \rightarrow z=z^{\prime}
\end{aligned}
$$

Axioms (2)-(4) show that methods, as understood here, are very detailed, they imply that two distinct forms with identical content (as in the case of a translation) must have been obtained via different methods. Also, methods can be combined: if $m$ and $m^{\prime}$ are methods, then their functional composition, $m^{\prime}(m(x))$, is also a method provided the $m(x)$ falls within the domain of $m$ '.

It is helpful to define a representation predicate as follows

$(D f 2)$ representation $(x)={ }_{\text {def }} \exists y, z, m$ representation $(x, y, z, m)$

It follows that the encoding relationship characterizes representations

$(T h 1)$ encodes_via $(x, y, m) \wedge v=x+y \rightarrow$ representation $(v)$

Whenever a representation exists, so does its form and content and so does the encoding method that binds them

$$
(A x 5) \text { representation }(x, y, z, m) \wedge \operatorname{Pre}(x, t) \rightarrow \operatorname{Pre}(y, t) \wedge \operatorname{Pre}(z, t) \wedge \operatorname{Pre}(m, t)
$$

A few observations are needed. First, we have not temporally characterized the encoding. This is a formal (atemporal) relationship. However, whenever the representation is present, so does the form, the content and the encoding. While the encoding does not depend on time, a form and a content are such in so far as they satisfy a suitable encoding relationship. Second, it is not possible for a form to exist without some content associated with it, and vice versa. Third, the same form (content) can be associated with different contents (forms) provided each encoding is done with a different method. Finally, we do not make any commitment on the possibility of form, content and method to disappear, i.e., if they cease to exist at some point or for some reason. The theory is neutral on these aspects. 
Representation, form and content exist in time, but neither is located in space because they are semi-abstract.

$$
\begin{aligned}
(A x 6) \text { representation }(x) & \vee \text { form }(x) \vee \operatorname{content}(x) \\
\rightarrow & \rightarrow \operatorname{Pr}(x, t) \wedge \forall y, t^{\prime} \neg \operatorname{Located}\left(x, y, t^{\prime}\right)
\end{aligned}
$$

Given a representation $x$, for simplicity and when there is no risk of confusion, we write $x_{f}$ for the representation form and $x_{c}$ for the representation content. A representing thing is the sum of a representation and a representation medium (the material support) which is a realization of the representation form

$$
\begin{array}{r}
(D f 3) \text { representing_thing }(r t, x, y)=\text { def }_{\text {representation_medium }}(x) \\
\wedge \text { representation }(y) \wedge r t=x+y \wedge \text { form_realization }\left(x, y_{f}\right)
\end{array}
$$

The entity representing thing is problematic in several foundational ontologies since it is the sum of a physical and a non-physical entity. In Dolce it is classified as Arbitrary Sum, in Yamato as Independent Entity. In an ontology that does not allow this kind of entities, one could model them indirectly as dependences across physical and semi-abstract entities.

$(A x 7)$ representation_medium $(x) \rightarrow$ physical_object $(x)$

(Ax8) form_realization $(x, y)$

$$
\begin{aligned}
& \rightarrow \text { representation_medium }(x) \wedge \text { form }(y) \\
& \wedge \exists \operatorname{rvm}(\text { representation }(r, y, v, m) \wedge \operatorname{Part}(r, x))
\end{aligned}
$$

(Ax9) form_realization $(x, y) \wedge$ representation $(r, y, u, m) \wedge \operatorname{Pre}(x, t)$

$$
\rightarrow \operatorname{Pre}(r, t)
$$

Then, we define the realization of content, as in the case of a procedure, as follows (here we use the standard satisfaction relation satisfies $(x, y)$ to mean that the entity $x$ satisfies the specification $y$ ).

$$
\begin{gathered}
(D f 4) \text { content_realization }(u, v)=d_{\text {def }}(\text { physical_object }(u) \vee \operatorname{event}(u)) \\
\wedge \operatorname{content}(v) \wedge \operatorname{satisfies}(u, v)
\end{gathered}
$$

We now concentrate on the case of sentences in natural language. As we have seen in the previous section, this case is particularly rich and complex to model. Here we aim to give a formal description of the basic elements of the theory. To model the realization of a form (form-realization) we need to refer to: representation, ink-mark, ink-pattern and pattern. The pattern is what a writer chooses when producing the representing-thing, say a manuscript or a printed book, and it corresponds to the font used in printing. (Here we do not attempt to characterize the pattern itself.) There are a few relationships that coordinate the dependences between representation and representing thing in (written) expressions of natural language: ink_pattern and pattern_of. We write ink_pattern $(x, y)$ to mean that $x$ is a pattern to be realized by a marking substance like ink (yet, in practice it can be electronic) of a representation form $y$. The pattern itself is semi-abstract. We write pattern_of $(x, z)$ to mean that $x$ is the pattern realized by the mark $z$ (of ink, in this case).

$(A x 10)$ ink_pattern $(x, y) \rightarrow \operatorname{pattern}(x) \wedge$ form $(y)$

$(A x 11) \operatorname{pattern}(x) \rightarrow \exists t \operatorname{Pre}(x, t) \wedge \forall y, t^{\prime} \neg \operatorname{Located}\left(x, y, t^{\prime}\right)$

$(A x 12)$ pattern_of $(x, y) \rightarrow \operatorname{pattern}(x) \wedge \operatorname{ink} \_\operatorname{mark}(y)$

$(A x 13)$ ink_mark $(x) \rightarrow$ physical_object $(x)$ 
Given these relationships, we can define the realization of a form in natural language (NLform_realization) which must constrain the relationships between the form, the pattern and the mark.

$$
\begin{aligned}
& \text { (Df5) NLform_realization }(u, v)={ }_{\text {def }} \text { form_realization }(u, v) \\
& \wedge \exists p, m \text { ink_pattern }(p, v) \wedge \operatorname{ink\_ mark}(m) \wedge \text { pattern_of }(p, m) \\
& \wedge \operatorname{part}(m, u)
\end{aligned}
$$

Remaining on the case of natural language, one could go further and define a sequence of symbols (letters of the alphabet) associated with a form. From this, it is possible to give the notion of sentence as a sequence of symbols that satisfies the language specification (the grammar), call it sentence_as_form. Then, we can define the representation of a sentence with this formula

$$
\begin{aligned}
& \text { (Df6) sentence_representation }(r)={ }_{\text {def }} \text { representation }(r) \\
& \wedge \text { sentence_as_form }\left(r_{f}\right) \wedge \exists z \operatorname{method}(z) \wedge \text { encodes_via }\left(r_{f}, r_{c}, z\right)
\end{aligned}
$$

To turn a symbolic representation into a representing thing, which we remind the readers counts as a form-realization rather than a content-realization, one first fixes the font and its size, so that the ink patterns are determined (these are realized as ink marks on a sheet of paper when printed). A symbolic representation is thus a sequence of letters' identifiers which are not physical entities. However, in practice a representation is created directly as a representing thing. The need of finer distinctions as introduced here raises when one aims to understand the phenomena from the ontological viewpoint.

\subsection{On Letters}

Here we apply the theory to clarify the nature of letters (characters) seen as representations. A letter " $a$ " written or printed on a sheet of paper is a tangible $2 \mathrm{D}$ object, a linear drawing which people recognize because the linear drawing encodes the commonly shared pattern "a". Leaving aside the specificity of the pattern and how it may evolve in time, we aim to uncover its contribution to the ontology of representation.

A letter's form encodes a pattern which can be the standard pattern of the letter, say, the pattern of letter "a". What is encoded in the letter form in addition to the standard pattern can be something else, namely, the letter as the first element of the alphabet. The essence of the pattern "a" is that it denotes the first letter of the alphabet. These two things should not be confused. Imagine replacing ' $x 1$ ' for ' $a$ ', ' $x 2$ ' for ' $b$ ', and so on. While computers can promptly process the new obtained sentences, this can be hard for humans. This failure (or serious difficulty) is caused by the lack of an encoded association between the new pattern and the identity of the letter of the alphabet as the content of the representation of " $a$ ". The association between ' $\mathrm{x} 1$ ' and the alphabet letter ' $a$ ' must be recreated with practice. Of course, the letter as a representation object is not influenced by the replacement and the consequent change in the association.

To conclude, all the many printed letters, say, "a", "a" and " $A$ " that we perceive in daily life are realizations rather than instances of letter "a". The linear drawing of a letter realizes a pattern of the letter. The realization is a form-realization. At the same time, it realizes the essential pattern of the letter since we do recognize the letter rather than a mere drawing. Thus, this is a content-realization. These observations show that when facing printed letters we are dealing with two distinct specifications. The former specification is the pattern of the written letter, the latter one is the standard (essential) 
pattern of the letter as an element of the alphabet. These two realizations produce one single (material) linear drawing.

\subsection{Formalization of Letter as Representation}

Now we can further specialize our previous theory relatively to expressions using letters. First, let us write classifies $(x, y)$ for the standard relationship $x$ classifies $y$; specifies $(x, p)$ for $x$ is a specification of pattern $y$; letter_pattern $(x)$ for the predicate that holds for the standard pattern of a letter; linear_drawing $(x)$ for the predicate that holds for the pattern that is realized; and id_alphabet $(n, x)$ for the relationship that identifies the pattern of the $n$-th letter of the alphabet. We write encodes_letter $(x, y, z)$ to mean an encoding $(z)$ occurring in letter representations in which the content $(y)$ classifies the form $(x)$.

\section{$(A x 14)$ encodes_letter $(x, y, z) \rightarrow$ classifies $(y, x) \wedge$ encodes_via $(x, y, z)$}

We can then say that the form of a letter is a specification of a pattern and that the content of a letter is either the letter of the alphabet or the standard pattern of the letter (here $n$ is an integer ranging from 1 to the number of letters in the alphabet, which is a finite number).

$$
\begin{aligned}
& \left(\text { Ax15) letter_form }(x) \rightarrow \exists p \text { specifies }(x, p) \wedge \operatorname{linear} \_d r a w i n g(p) \wedge \operatorname{form}(x)\right. \\
& (D f 7) \text { letter_content }(x)={ }_{\text {def }} \text { content }(x) \wedge \exists n, p(\text { id_alphabet }(n, p) \\
& \vee(\operatorname{specifies}(x, p) \wedge \text { letter_pattern }(p)))
\end{aligned}
$$

Finally, we can define a letter representation

$$
\begin{gathered}
\text { (Df8) letter_representation }(r, x, y, z)={ }_{\text {def }} \text { representation }(r, x, y, z) \\
\wedge \text { letter_form }(x) \wedge \text { letter_content }(y) \\
\wedge \text { encodes_letter }(x, y, z)
\end{gathered}
$$

An ontological difficulty that led to this theory is due to contrasting properties of letters: letters are at the same time symbolic and analogue entities. By distinguishing the symbolic aspect (the element in the alphabet) from the analogue aspect (the letter pattern), we obtain a coherent representation theory which can clarify their relationship. Accordingly, letters as form of a symbolic representation are dealt with as identifiers of entities of the alphabet, letters in the form of representing thing are dealt with as analogue representations whose form is a linear drawing. As the formalization shows, what appears in the form of a symbolic representation is the letter as the content, i.e., the identifier of the letter in the alphabet.

\section{Discussion}

\subsection{Classifying Representation Entities}

In Table 1 we summarize the entities we have discussed addressing the ontology of representation. Novel has no direct physical entity in the real world. There are corresponding physical entities that are realizations like books, eBooks etc., that is, representing things. The other representations are associated with two types of physical entities. This difference comes from the fact that a representation which has a designed content has two ways of realization: one realization is a product according to the specification (content-realization) and the other is a product according to the form (formrealization). 
Table 1 Summary of representational entities.

\begin{tabular}{|l|l|l|l|l|}
\hline & \multicolumn{1}{|c|}{ Representation } & \multicolumn{1}{|c|}{ Content } & \multicolumn{1}{|c|}{$\begin{array}{c}\text { Representing thing } \\
\text { (form-realization) }\end{array}$} & $\begin{array}{c}\text { Individual as } \\
\text { content- } \\
\text { realization }\end{array}$ \\
\hline $\begin{array}{l}\text { Industrial } \\
\text { Product }\end{array}$ & $\begin{array}{l}\text { Its design drawing/ } \\
\text { specification }\end{array}$ & $\begin{array}{l}\text { The specification of } \\
\text { the structure/functions }\end{array}$ & $\begin{array}{l}\text { The printed design } \\
\text { drawing/specification }\end{array}$ & Itself \\
\hline Painting & $\begin{array}{l}\text { The digitized image } \\
\text { of the painting } \\
\text { displayed on a screen }\end{array}$ & $\begin{array}{l}\text { What is painted such as } \\
\text { persons, scenery... }\end{array}$ & $\begin{array}{l}\text { The painting on a } \\
\text { canvas }\end{array}$ & None \\
\hline Music & The musical score & $\begin{array}{l}\text { The specification of } \\
\text { the sound sequence }\end{array}$ & $\begin{array}{l}\text { The printed musical } \\
\text { score }\end{array}$ & $\begin{array}{l}\text { The sound } \\
\text { sequence }\end{array}$ \\
\hline Procedure & $\begin{array}{l}\text { The description of } \\
\text { the procedure }\end{array}$ & $\begin{array}{l}\text { The specification of } \\
\text { the actions/operations } \\
\text { Letter }\end{array}$ & $\begin{array}{l}\text { The printed description } \\
\text { of the procedure }\end{array}$ & $\begin{array}{l}\text { The execution } \\
\text { process of } \\
\text { procedure }\end{array}$ \\
\hline Novel/book & $\begin{array}{l}\text { The text (the novel } \\
\text { one writes })\end{array}$ & $\begin{array}{l}\text { The specification of its } \\
\text { standard pattern }\end{array}$ & $\begin{array}{l}\text { The letter written on a } \\
\text { theet of paper }\end{array}$ & $\begin{array}{l}\text { The linear } \\
\text { drawing }\end{array}$ \\
\hline
\end{tabular}

Table 1 tells us that the representing thing (the form-realization) of a painting is the "painting on a canvas", what we usually see in a museum or art exposition. The representation that corresponds to it must be a specification of the form encoding the painting content. Like in the case of letter, the form of a painting is the specification of the "ink pattern", and the representation of a painting is the digitized image of the painting displayed on a screen. In this sense, painting is special: it is primarily what it shows, i.e., the form. In this it is like handwriting in calligraphy, essentially the form that it manifests. On the contrary, novel has both a visible aspect (ink-patterns/marks of letters and sentences) and a non-visible aspect, its content (the story).

As we already discussed, entities obtained by the form-realization and the contentrealization of a letter coincide with each other in reality. In the table, we distinguish them by using different expressions to reflect the differences of the realization processes: "letter written on a sheet of paper" for the former and "linear drawing" for the latter.

\subsection{On (the Missing) Content}

Precisely speaking, sentences have two contents: the meaning of the sentence and the style (beauty) of the sentence. The discussion thus far only deals with the former content. To make the discussion more comprehensive, the latter content needs to be incorporated in the theory. A good writer has his/her own style of sentences which is the heart of writing. In this view, a natural language expression which is classified into the representation form has also another content, we may call it style-content, which is the specification of his/her sentence style. This style-content requires its specific relationships but does not need further discussions as the formalization is essentially similar by the machinery already developed for the meaning of a sentence (see also [3]).

\subsection{Copying and Identity}

Given a representation whose form is a sequence of symbols, one can copy the sequence of symbols or copy the sequence of the symbols' images (photocopying). In both cases, what one copies is the representing thing. Since symbols are unique, one cannot really copy just symbols: one copies a representing thing which is a realization of symbols. Copying is thus the realization of the same representation on a different medium. The issue is what "same" means. Roughly speaking, here two kinds of sameness are present. 
One is given by a representing thing that realizes the representation form of another representing thing via different fonts or handwritings. Here sameness means that it is a realization of the same form (thus, the very same symbols). The other kind of sameness corresponds to an act of photocopying, that is, to obtain a representing thing that realizes the representation form of another representing thing via the same ink patterns (the ink marks of the two representing things have the same pattern).

The distinction between copying and photocopying tells us about relative identity of representations and of representing things. There are four levels of identity to keep in mind: (0) content level, (1) symbol level, (2) ink pattern level and (3) medium level. A perfect translation using different languages, say from a logical theory to an equivalent theory formulated in a different logical language, is a copy at the level (0). These entities are identical with respect to content. A hand-written copy of a sentence on a sheet of paper is identical to the original one at the level (0) and (1), as they realize the same symbols. A photocopy of a sentence on a sheet of paper is identical to the original one at the level (0), (1) and (2), as they realize the same patterns (modulo the quality of the photocopy). At the medium level, two physical entities cannot be identical as we assume that the medium in the two representation things must be different.

\section{Related Work}

A comprehensive state of the art on informational objects has been presented recently [12]. Philosophy of language and thought [14] (and so approaches in natural language understanding and processing), treats syntactic expressions (the form in our term) and meaning (the content in our term) as different entities to separate syntactic and semantic analyses as far as possible. In our ontology of representation, the form and the content constitute a single entity, called representation, due to an encoding relationship. This is mainly because the aim of our theory is to provide an ontological understanding of a "common-sense view of the world" rather than tools for natural language processing/ understanding.

The ontology of literary works discussed in philosophy by Thomasson [13] takes the view that a particular sound sequence is not an instance of a class. Our theory follows this intuition and adds that this sequence should be understood as a realization of a piece of music. Thomasson discusses neither specification nor our development of the symbols/ letters relationships. Also, conventional ontologies of literary works [12] [13] concentrate on how readers (users in our term) interpret/understand the works. Our ontology does not since we concentrate on foundational aspects which are domain independent. We support the idea that, ontologically, any representation including literary works exists independently of its readers/users.

One of the unsolved issues in ontology of literary works is whether all literary works share the same ontological status [13]. In fact, people tend to believe that novels are abstract objects and paintings are concrete objects. Our ontology gives a comprehensive view to this issue by providing a notion, that of representation, that subsumes novels, poems, paintings, and sculptures.

Since this paper aims to develop a refined theory of information objects as discussed in applied ontology, perhaps the main theory to discuss is the Information Artifact Ontology (IAO) approach presented in [1][2]. Even though strictly related, IAO and our theory focus on different entities. The former discusses information, the latter centers on representations and takes information to be a role played by a representation under the context of an informing activity. 
The term Information Content Entity (ICE), the main type modeled in IAO, turns out to be ambiguous when analyzed from our theory's viewpoint. A terminology match can help to clarify this. Content in ICE combines both content and form in our terminology. IQE, which is a concretization of ICE, corresponds to representing thing in our theory. Although some IQEs are said to be concretization of ICE, examples of ICE are not given except in the following excerpt from [2]:

...for example, the dependent entity which is the pattern of ink marks in your copy of the novel War and Peace (a complex quality in BFO terms) - are able to migrate from one bearer to another (e.g., through use of a photocopier).

This piece suggests that the dependent entity made of the ink patterns is an ICE because it satisfies the definition of ICE. This does not seem to follow from the guidelines of the IAO approach. An ICE is what is ready to be concretized in a representing thing (in our terminology) and has strong association with how to achieve the concretization as seen in "this sentence is concretized in this pattern of ink marks on this piece of paper" [2]. That is, the definition of ICE covers not only content but also content encoded in form (the above example). Hence the shapes/patterns of letters seem to be embedded in sentences. In our theory of representation, on the other hand, the separation is clearly made as the duality of letters is explicitly modeled: letters appear as identifiers in sentences and are analogue representations in themselves. A sentence in our theory, in the sense of representation form, is composed not of specific letters of specified fonts which explicitly have their own "ink pattern" but of identifiers of letters independently of the "ink pattern". In short, a sentence in IAO is ink-pattern-dependent, while in our theory it is not. This might be mainly due to the so-called realist approach [9] of the underlying ontology and the emphasis on representing thing-centered entities.

\section{Concluding Remarks}

Representation, in our view, is more fundamental than information and more approachable for ontological analysis. Because of this, here we studied ontological representation objects (ORO), their ontological nature and an initial axiomatization. There are three types of content: denotation, specification and description. We focused on specification which characterizes representation where there are performers as content-realizers, as well as creators, like in procedures and pieces of music. A clear separation between representation (ORO) and representing thing enabled us to identify the ontological status of a book and of what one writes beyond the common yet naïve separation between content and material support. The fact that what one writes is fontindependent shows that a book one writes is an ORO rather than a representing thing.

This paper presents only the basics of an ontology of representation and much is left unexplored. They plan to expand this work towards a unified theory of representation that includes a full explanation of how to model letters, contribute to move towards a more solid ontological foundation of information objects like data, information and knowledge.

\section{References}

[1] Ceusters W. An information artifact ontology perspective on data collections and associated representational artifacts. Stud Health, Technol Inform. 2012; 180: 68-72.

[2] Ceusters W, Smith B. Aboutness: Towards Foundations for the Information Artifact Ontology. Proc. of the Sixth International Conference on Biomedical Ontology (ICBO); 2015 (CEUR 1515). 
[3] Mizoguchi R. Advanced course of ontological engineering Part 3, Section 2 Ontology of representation. New Generation Computing; 2004; 22 (2): 193-220.

[4] Mizoguchi R. YAMATO: yet another more advanced top-level ontology. Proceedings of the Sixth Australasian Ontology Workshop; 2010: 1-16.

[5] Yablo S. Aboutness. Princeton University Press. 2014.

[6] Zins C. Conceptual approaches for defining data, information, and knowledge. Journal of the American society for information science and technology. 2007; 58(4): 479-493.

[7] Lewis D. Convention: A philosophical study. John Wiley \& Sons; 2008.

[8] Ni P. Moral and philosophical implications of Chinese calligraphy. Grand Valley Review. 1999; 20.1.

[9] Smith B, Ceusters W. Ontological realism: A methodology for coordinated evolution of scientific ontologies, Applied Ontology. 2010; 5 (3):139-188.

[10] Mizoguchi R, Sunagawa E, Kozaki K, Kitamura Y. The model of roles within an ontology development tool: Hozo, Applied Ontology. 2007; 2 (2), 159-179.

[11] Mizoguchi R, Galton A, Kitamura Y, Kozaki K. Families of roles: A new theory of occurrent-dependent roles, Applied Ontology. 2015; 10 (3-4), 367-399.

[12] Sanfilippo EM. Ontologies for information entities: State of the art and open challenges. Applied ontology. $2021 ; 16(2): 111-135$.

[13] Thomasson AL. The ontology of literary works. In The Routledge Companion to Philosophy of Literature. 2016; Routledge; 349-358.

[14] Dummett M. Frege: Philosophy of Language. Harvard University Press. 2nd edition. 1981. 